\title{
Reversible posterior leukoencephalopathy syndrome during sunitinib therapy for metastatic renal cell carcinoma
}

\author{
JAMAL OULAD HADJ $^{1}$, ROGIER DEN BRAVEN ${ }^{2}$, CORRINE TILLIER ${ }^{1}$, HANS M. SCHRIJVER ${ }^{2}$, \\ HENK M.W. VERHEUL ${ }^{1}$ and HANS J. VAN DER VLIET ${ }^{1}$ \\ ${ }^{1}$ VU University Medical Center, Department of Medical Oncology, 1081 HV Amsterdam; \\ ${ }^{2}$ Westfriesgasthuis, Department of Neurology, 1624 NP Hoorn, The Netherlands
}

Received January 5, 2012; Accepted March 13, 2012

DOI: $10.3892 / \mathrm{ol} .2012 .646$

\begin{abstract}
Sunitinib is an oral receptor tyrosine kinase inhibitor with potent antiangiogenic and antitumor activity that is approved for the treatment of advanced renal cell carcinoma (RCC), malignant gastrointestinal stromal tumors and pancreatic neuroendocrine tumors. Well-known side effects of sunitinib include hypertension, fatigue, thyroid dysfunction, cardiotoxicity, gastrointestinal toxicity and skin toxicity. In this study, we report the case of a 61-year-old male with papillary metastatic RCC who responded to sunitinib but developed generalized tonic-clonic seizures during the third cycle. Magnetic resonance imaging (MRI) was compatible with reversible posterior leukoencephalopathy syndrome (RPLS). After the administration of anti-epileptic drugs and the withdrawal of sunitinib there was rapid clinical improvement. Notably, radiological characteristics of RPLS persisted during second-line therapy with the mammalian target of rapamycin (mTOR) inhibitor everolimus and only resolved when everolimus was terminated due to disease progression. Although sunitinib-induced RPLS has been reported previously, our case is the first to additionally suggest that everolimus may sustain and therefore potentially contribute to the occurrence of RPLS.
\end{abstract}

\section{Introduction}

Kidney tumors comprise $2 \%$ of all adult malignancies and account for approximately 102,000 mortalities worldwide annually (1). Renal cell carcinoma (RCC) constitute the majority of all kidney tumors and can be classified histologically as clear cell (60-80\%), papillary (10-15\%), chromophobe $(5-10 \%)$ and collecting duct carcinoma $(<1 \%)$. Approximately $30 \%$ of all patients with RCC have metastatic disease at

Correspondence to: Dr Hans J. van der Vliet, Department of Medical Oncology, VU University Medical Center, De Boelelaan 1117, 1081 HV Amsterdam, The Netherlands

E-mail: jj.vandervliet@vumc.nl

Key words: renal cell cancer, sunitinib, reversible posterior leukoencephalopathy syndrome presentation and $\sim 50 \%$ of patients undergoing curative surgery are expected to experience relapse at distant sites $(2,3)$.

The treatment of metastatic (m)RCC has markedly changed over the last 5 years due to the antitumor efficacy of two groups of targeted agents, i.e., agents that inhibit vascular endothelial growth factor (VEGF)-signaling pathways and those that inhibit mammalian target of rapamycin (mTOR) (4). For example, the VEGF-receptor/c-kit/platelet-derived growth factor receptor/FMS-like tyrosine kinase 3 tyrosine kinase inhibitor sunitinib, exhibits a $31 \%$ partial response rate in mRCC patients, disease stabilization in an additional $48 \%$ of $\mathrm{mRCC}$ patients and an increase in median progression-free survival from 5 to 11 months (5). For patients with mRCC that progressed to VEGF receptor tyrosine kinase inhibitor therapy, the orally administered mTOR inhibitor everolimus was recently shown to prolong progression-free survival relative to placebo from 1.9 to 4.9 months $(\mathrm{p}<0.001)$, providing an important additional therapeutic tool for this patient category $(6,7)$.

Well-known side-effects of sunitinib include hypertension, fatigue, thyroid dysfunction, cardiotoxicity, gastrointestinal toxicity and skin toxicity (8). In this study, we report the case of a 61-year-old male with papillary mRCC who developed reversible posterior leukoencephalopathy syndrome (RPLS) during sunitinib therapy. Our case is the seventh patient reported in the literature (9) whose treatment with sunitinib was complicated by RPLS, but the first to additionally suggest that everolimus may prolong radiological alterations characteristic of RPLS. Patient consent was obtained for this case report.

\section{Case report}

A 61-year-old male with a history of hypercholesterolemia, hypertension and type 2 diabetes was referred to our outpatient clinic following diagnosis with mRCC. Due to hematuria, flank pain, weight loss and a renal mass, the patient had undergone a right-sided nephrectomy with lymph node dissection three months earlier. Histopathological examination revealed a T3bN2Mx papillary cell RCC. Besides a non-productive cough and mild shortness of breath on exertion, the patient had no specific complaints. Physical examination revealed no relevant abnormalities; the patient had a WHO performance score of 1 and a blood pressure of 150/88 $\mathrm{mmHg}$. A mild 

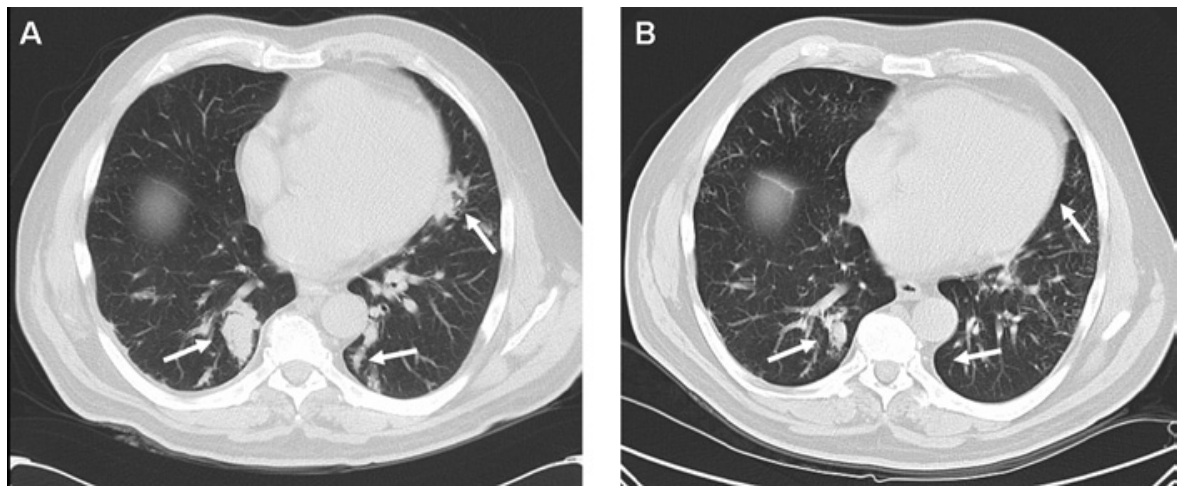

Figure 1. CT-scan of the chest (A) before and (B) after two cycles of sunitinib. Arrows indicate areas of tumor localization.
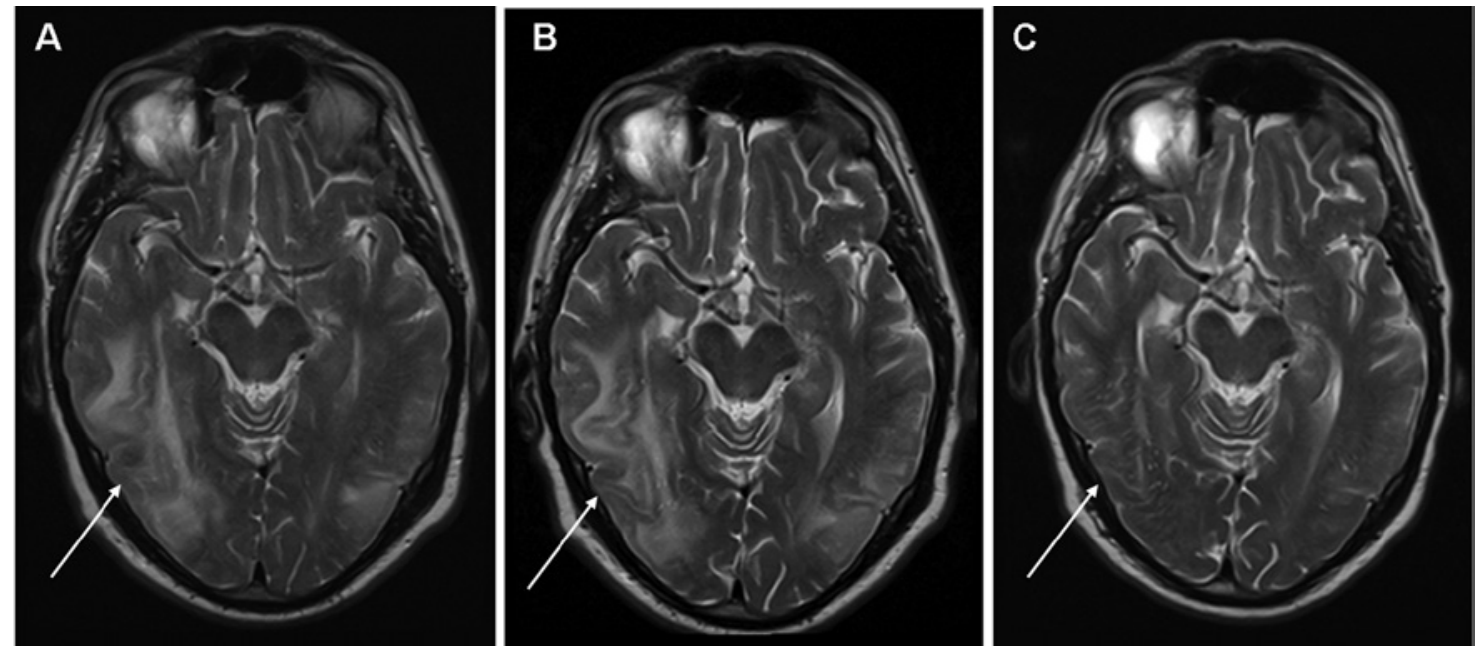

Figure 2. MRI-scan (T2-weighted images) of the brain during sunitinib therapy at (A) presentation with RPLS, (B) during everolimus therapy (six weeks after sunitinib was stopped) and (C) 2 weeks after cessation of everolimus. Extensive edema is evident in the right parieto-temporal, right parietal and left parietooccipital areas of the brain. RPLS, reversible posterior leukoencephalopathy syndrome.

anemia [hemoglobin $7.8 \mathrm{mmol} / \mathrm{l}$ (normal $8.5-11 \mathrm{mmol} / \mathrm{l})]$ and a mild renal insufficiency (glomerular filtration rate $45 \mathrm{ml} / \mathrm{min}$ ) were noted on laboratory evaluation. Serum levels of calcium and lactate dehydrogenase were normal. Computed tomography (CT) of the chest and abdomen demonstrated metastases in mediastinal and retroperitoneal lymph nodes, as well as bilateral pulmonary metastases.

Treatment with sunitinib (50 mg daily for 4 weeks every 6 weeks) was initiated. Although antitumor responses are more frequently observed in patients with clear cell as compared to papillary cell RCC (10), the patient responded clinically with a reduction in his non-productive cough and exertional shortness of breath, and radiologically with a partial response of pulmonary metastases (Fig. 1). Treatment was well tolerated with no alterations in renal function, blood pressure, thyroid hormone function and no hematological toxicity.

During the third week of the third cycle of sunitinib, the patient presented to the emergency department following an episode of absence and a generalized tonic-clonic seizure. Physical and neurological examination revealed no other abnormalities apart from hypertension $(202 / 101 \mathrm{mmHg}$ ). The epileptic seizure responded well to an initial combination of midazolam (7.5 mg i.v.) and phenytoin (1250 mg i.v.).
Laboratory tests, including renal function and serum electrolytes, showed no significant changes. A CT scan of the brain revealed hypodense areas suggestive of edema but did not rule out metastases. Subsequent magnetic resonance imaging (MRI) of the brain was compatible with RPLS with extensive edema predominantly in the right parieto-temporal, right parietal and left parieto-occipital areas of the brain with no evidence of cerebral metastases (Fig. 2A). Treatment with sunitinib was stopped and anti-epileptic treatment consisting of oral sodium valproate $(750 \mathrm{mg}$ bid) was initiated and the patient made a further uneventful recovery. The increased blood pressure that was noted upon presentation rapidly returned to normal and the patient's regular antihypertensive treatment consisting of the combination of a thiazide diuretic, an ACE-inhibitor and a calcium antagonist was continued.

Four weeks later, a CT scan showed progressive disease of pulmonary metastases and retroperitoneal lymphadenopathy accompanied by a subjective increase in exertional shortness of breath, non-productive cough and short episodes of upper abdominal pain. Second-line treatment with oral everolimus (10 mg once daily) was initiated. Although the treatment was well tolerated, no symptomatic improvement in pulmonary complaints occurred and treatment with everolimus was 
terminated after 4 weeks due to progressive disease. Notably, a follow-up MRI scan of the brain performed in the second week of everolimus therapy ( 6 weeks after sunitinib treatment was terminated) still showed radiological evidence of RPLS (Fig. 2B). A repeat MRI of the brain performed 2 weeks following cessation of everolimus showed complete resolution of RPLS (Fig. 2C). Palliative treatment was provided by the patient's general practitioner. The patient succumbed to the disease 6 months later.

\section{Discussion}

RPLS is a noteworthy clinical and radiological entity that was first described by Hinchey et al (11). Clinical symptoms frequently observed in patients with RPLS include epileptic seizures, altered mental status, vomiting, visual disturbances and headache. Mechanistically, RPLS corresponds to a cerebral vasogenic edema associated with a variety of conditions including arterial hypertension, eclampsia, collagen vascular disorders, Guillain Barre syndrome, thrombotic thrombocytic purpura, acute porphyria and postcarotid endarterectomy. However, it is most commonly caused by immunosuppressive and cytotoxic drugs including cyclosporin A, tacrolimus, cisplatin, cytarabine, intravenous immunoglobulins, L-asparaginase, 5-fluorouracil, interferon- $\alpha$, and more recently by anti-angiogenic agents including bevacizumab, sorafenib and sunitinib (9,11-20). Typical MRI findings in patients with RPLS include edema involving the white matter, predominantly in the posterior portions of the cerebral hemispheres, particularly bilaterally in the parieto-occipital regions, although other areas of the brain may also be affected $(11,17)$.

Although the exact pathogenesis of RPLS remains unclear, it is most likely due to a disruption of cerebral vascular endothelial cells and impaired cerebrovascular autoregulation leading to edema (11). During hypertensive periods the upper limit of cerebral autoregulation, which normally maintains a constant perfusion rate during blood pressure changes, is exceeded resulting in hyperperfusion and the formation of vasogenic edema of the brain (21). Furthermore, renal dysfunction appears to predispose patients to the development of RPLS, likely due to fluid overload (16). Cytotoxic drugs may have direct toxic effects on the vascular endothelium leading to capillary leakage and cerebral edema, even in normotensive patients using drugs in normal therapeutic ranges $(11,22,23)$. Angiogenesis inhibitors, including sunitinib, may trigger RPLS through the induction of endothelial dysfunction and/or hypertension (9,12-14). In our patient, there was pre-existing hypertension and mild renal dysfunction. Although his blood pressure was well controlled during the first cycles of sunitinib, an increase was observed when the patient was admitted with RPLS. This increased blood pressure rapidly normalized after the initiation of anti-epileptic treatment and the discontinuation of sunitinib. Notably, radiological alterations compatible with RPLS persisted during second-line therapy with everolimus. We believe that everolimus may have contributed to this lengthy (>42 days) persistence of radiological abnormalities, as these rapidly resolved when everolimus was discontinued. Additionally, other authors have demonstrated that radiological signs of RPLS normally improve with a median of 20 days in $88 \%$ of patients, with complete or near-complete resolution in
$70 \%$ of patients (17). RPLS has not been reported in association with everolimus or other mTOR inhibitors. As mTOR inhibitors are believed to exert antitumor effects by direct inhibition of tumor cell growth and proliferation, as well as the inhibition of angiogenesis (via inhibition of tumor cell VEGF production and VEGF-induced proliferation of endothelial cells), the latter mechanism may contribute to or sustain RPLS (24).

Our case is the seventh report in the literature of RPLS associated with sunitinib, and the first to additionally suggest that everolimus sustains and therefore potentially contributes to the occurrence of RPLS. Early recognition of RPLS is critical as neurological damage may be irreversible if RPLS is not treated immediately by adequate blood pressure regulation and the discontinuation of any causative drugs, as permanent neurological damage may result from cerebral infarction or hemorrhages $(25,26)$.

\section{References}

1. Parkin DM, Bray F, Ferlay J, et al: Estimating the world cancer burden: Globocan 2000. Int J Cancer 94: 153-156, 2000.

2. van der Veldt AA, Haanen JB, van den Eertwegh AJ, et al: Targeted therapy for renal cell cancer: current perspectives. Discov Med 10: 394-405, 2010.

3. Linehan WM, Zbar B, Bates SE, et al: Cancer of the kidney and ureter In: Cancer: Principles and Practice of Oncology. 6th edition. DeVita VT Jr, Hellman S and Rosenberg SA (eds). Lippincott Williams \& Wilkins, Philadelphia, pp1362-1396, 2001.

4. Powles T, Chowdury S, Jones R, et al: Sunitinib and other targeted therapies for renal cell carcinoma. Br J Cancer 104: 741-745, 2011.

5. Motzer RJ, Hutson TE, Tomczak P, et al: Sunitinib versus interferon alfa in metastatic renal-cell carcinoma. $\mathrm{N}$ Engl J Med 356: 115-124, 2007.

6. Motzer RJ, Escudier B, Oudard S, et al: Efficacy of everolimus in advanced renal cell carcinoma: a double-blind, randomised, placebo-controlled phase III trial. Lancet 372: 449-456, 2008.

7. Motzer RJ,Escudier B, Oudard S, et al: Phase 3 trial of everolimus for metastatic renal cell carcinoma. Cancer 116: 4256-4265, 2010.

8. Verheul HM and Pinedo HM: Possible molecular mechanisms involved in the toxicity of angiogenesis inhibition. Nat Rev Cancer 7: 475-485, 2007.

9. Padhy BM, Shanmugam SP, Gupta YK, et al: Reversible posterior leucoencephalopathy syndrome in an elderly male on sunitinib therapy. Br J Clin Pharmacol 71: 777-779, 2011.

10. Choueiri TK, Plantade A, Elson P, et al: Efficacy of sunitinib and sorafenib in metastatic papillary and chromophobe renal cell carcinoma. J Clin Oncol 26: 127-131, 2008.

11. Hinchey J, Chaves C, Appignani B, et al: A reversible posterior leukoencephalopathy syndrome. N Engl J Med 334: 494-500, 1996.

12. Martín G, Bellido L and Cruz JJ: Reversible posterior leukoencephalopathy syndrome induced by sunitinib. J Clin Oncol 25: $3559,2007$.

13. Medioni J, Cojocarasu O, Banu E, et al: Reversible encephalopathy syndrome secondary to sunitinib for metastatic renal cell carcinoma patient. Targ Oncol 2: 193-195, 2007.

14. Cumurciuc R, Martinez-Almoyna L, Henry C, et al: Posterior reversible encephalopathy syndrome during sunitinib therapy. Rev Neurol (Paris) 164: 605-607, 2008.

15. Chen A and Agarwal N: Reversible posterior leucoencephalopathy syndrome associated with sunitinib. Intern Med J 39: 341-342, 2009.

16. Kapiteijn E, Brand A, Kroep J, et al: Sunitinib induced hypertension, thrombotic microangiopathy and reversible posterior leukencephalopathy syndrome. Ann Oncol 18: 1745-1747, 2007.

17. Fugate JE, Claassen DO, Cloft HJ, et al: Posterior reversible encephalopathy syndrome: associated clinical and radiologic findings. Mayo Clin Proc 85: 427-432, 2010.

18. Govindarajan R, Adusumilli J, Baxter DL, et al: Reversible posterior leukencephalopathy syndrome induced by RAF kinase inhibitor BAY 43-9006. J Clin Oncol 24: e48, 2006. 
19. Glusker P, Recht L and Lane B: Reversible posterior leukoencephalopathy syndrome and bevacizumab. N Engl J Med 354: 980-981, 2006.

20. Ozcan C, Wong SJ, and Hari P: Reversible posterior leukencephalopathy syndrome and bevacizumab. N Engl J Med 354: 981-982, 2006.

21. Paulson OB, Strandgaard S and Edvinsson L: Cerebral autoregulation. Cerebrovasc Brain Metab Rev 2: 161-192, 1990.

22. Covarrubias DJ, Luetmer PH and Campeau NG: Posterior reversible encephalopathy syndrome: prognostic utility of quantitative diffusion-weighted MR images. AJNR Am J Neuroradiol 23: 1038-1048, 2002.
23. Ito Y, Arahata Y, Goto Y, et al: Cisplatin neurotoxicity presenting as reversible posterior leukoencephalopathy syndrome. AJNR Am J Neuroradiol 19: 415-417, 1998.

24. Agarwala SS and Case S: Everolimus (RAD001) in the treatment of advanced renal cell carcinoma: a review. Oncologist 15: 236-245, 2010

25. Schwartz RB: A reversible posterior leukencephalopathy syndrome. N Engl J Med 334: 1743, 1996.

26. Weingarten K, Barbut D, Fillipi C, et al: Acute hypertensive encephalopathy: findings on spin-echo and gradient-echo MR-imaging. Am J Roentgenol 162: 665-670, 1994. 